\section{OPEN ACCESS}

Edited by: Chantal Michel,

University of Bern, Switzerland

Reviewed by:

Danilo Assis Pereira,

Instituto Brasileiro de

Neuropsicologica e Ciencias

Cognitivas, Brazil

Rafael Penadés,

Hospital Clínic de Barcelona, Spain

${ }^{*}$ Correspondence:

Tina D. Kristensen

tina.dam.kristensen@regionh.dk

Specialty section:

This article was submitted to

Psychopathology,

a section of the journal

Frontiers in Psychiatry

Received: 13 April 2020 Accepted: 10 August 2020 Published: 28 August 2020

Citation:

Kristensen TD, Ebdrup BH, Hjorthøj C, Mandl RCW, Raghava JM, Jepsen JRM, Fagerlund $B$, Glenthøj LB, Wenneberg C, Krakauer K, Pantelis C, Glenthøj BY and Nordentoft M (2020) No Effects of Cognitive Remediation on Cerebral White Matter in Individuals at UItra-

High Risk for Psychosis - A

Randomized Clinical Trial.

Front. Psychiatry 11:873.

doi: 10.3389/fpsyt.2020.00873

\title{
No Effects of Cognitive
} Remediation on Cerebral White Matter in Individuals at Ultra-High Risk for Psychosis - A Randomized Clinical Trial

Tina D. Kristensen ${ }^{1,2 *}$, Bjørn H. Ebdrup ${ }^{2,3,4}$, Carsten Hjorthøj ${ }^{1,5}$, René C. W. Mandl ${ }^{2,6}$, Jayachandra M. Raghava ${ }^{2,7}$, Jens Richardt M. Jepsen ${ }^{2,8}$, Birgitte Fagerlund ${ }^{2,9}$, Louise B. Glenthøj ${ }^{1,2}$, Christina Wenneberg ${ }^{1,2}$, Kristine Krakauer $^{2}$, Christos Pantelis ${ }^{2,4}$, Birte Y. Glenthøj ${ }^{2,3}$ and Merete Nordentoft ${ }^{1,2,3}$

${ }^{1}$ Copenhagen Research Center for Mental Health, CORE, Mental Health Centre Copenhagen, University of Copenhagen, Hellerup, Denmark, ${ }^{2}$ Center for Clinical Intervention and Neuropsychiatric Schizophrenia Research, CINS, and Center for Neuropsychiatric Schizophrenia Research, CNSR, Mental Health Centre Glostrup, University of Copenhagen, Glostrup, Denmark, ${ }^{3}$ Department of Clinical Medicine, Faculty of Health and Medical Sciences, University of Copenhagen, Copenhagen, Denmark, ${ }^{4}$ Melbourne Neuropsychiatry Center, Department of Psychiatry, The University of Melbourne, Melbourne, VIC, Australia, 5 Section of Epidemiology, Department of Public Health, University of Copenhagen, Copenhagen, Denmark, ${ }^{6}$ Brain Center, University Medical Center Utrecht, Utrecht, Netherlands, ${ }^{7}$ Functional Imaging Unit, Department of Clinical Physiology, Nuclear Medicine and PET, University of Copenhagen, Glostrup, Denmark, ${ }^{8}$ Child and Adolescent Mental Health Centre, Mental Health Services, Capital Region of Denmark, University of Copenhagen, Hellerup, Denmark, ${ }^{9}$ Department of Psychology, Faculty of Social Sciences, University of Copenhagen, Copenhagen, Denmark

Background: Individuals at ultra-high risk for psychosis (UHR) present with subtle alterations in cerebral white matter (WM), which appear to be associated with clinical and functional outcome. The effect of cognitive remediation on WM organization in UHR individuals has not been investigated previously.

Methods: In a randomized, clinical trial, UHR individuals aged 18 to 40 years were assigned to treatment as usual (TAU) or TAU plus cognitive remediation for 20 weeks. Cognitive remediation comprised $20 \times 2$-h sessions of neurocognitive and social-cognitive training. Primary outcome was whole brain fractional anisotropy derived from diffusion weighted imaging, statistically tested as an interaction between timepoint and treatment group. Secondary outcomes were restricted to five predefined region of interest (ROI) analyses on fractional anisotropy, axial diffusivity, radial diffusivity and mean diffusivity. For significant timepoint and treatment group interactions within these five ROls, we explored associations between longitudinal changes in WM and cognitive functions/clinical symptoms. Finally, we explored dose-response effects of cognitive remediation on WM.

Results: A total of 111 UHR individuals were included. Attrition-rate was 26\%. The cognitive remediation group completed on average $12 \mathrm{~h}$ of neurocognitive training, which was considerably lower than per protocol. We found no effect of cognitive remediation on whole-brain FA when compared to treatment as usual. Secondary ROI analyses revealed a nominal significant interaction between timepoint*treatment of $A D$ in left medial 
lemniscus ( $P=0.016)$ which did not survive control for multiple comparisons. The exploratory test showed that this change in $A D$ correlated to improvements of mental flexibility in the cognitive remediation group $(p=0.001)$. We found no dose-response effect of neurocognitive training on WM.

Conclusions: Cognitive remediation comprising $12 \mathrm{~h}$ of neurocognitive training on average did not improve global or regional WM organization in UHR individuals. Further investigations of duration and intensity of cognitive training as necessary prerequisites of neuroplasticity-based changes are warranted.

Clinical Trial Registration: ClinicalTrials.gov, identifier NCT02098408.

Keywords: ultra-high risk for psychosis, cognition, white matter, clinical trial, cognitive remediation, diffusionweighted imaging

\section{INTRODUCTION}

Individuals at ultra-high risk (UHR) for psychosis present with cognitive impairments (1), intermediate between the marked cognitive deficits observed in patients with manifest psychotic disorders, and unaffected healthy controls (2-4). The cognitive impairments in UHR individuals are present across multiple domains (5), with small to large effect sizes compared to healthy controls (6).

Moreover, magnetic resonance imaging studies have revealed widespread, but subtle alterations in WM organization in UHR individuals, typically measured as lower fractional anisotropy (7). Fractional anisotropy (FA) is the currently most widely applied index of WM organization (8), sensitive to a broad range of neurobiological substrates, such as axonal density, axonal crosssection, myelin and crossing fibers (9). These WM-alterations have been associated with severity of cognitive deficits (10) and functional outcome (11), and appear to convey a liability for transition to frank psychosis (12). In a previous cross-sectional study of the current sample (10), we performed multivariate analyses of baseline data on associations between WM and cognition, comparing UHR individuals to healthy controls. At a whole-brain level, we identified an association between globally higher fractional anisotropy with a pattern of better cognitive functions in UHR individuals, but not in healthy controls. Furthermore, we identified cognitive functions associated with regional WM-interaction in five regions (fornix, medial lemniscus bilateral, left superior cerebellar peduncle, and left uncinate fasciculus) when comparing UHR individuals to healthy controls. The cognitive functions contributing reliably to the significant interactions of regional WM, were verbal intelligence, verbal fluency, planning, verbal working memory, and mental flexibility (10). The results suggested, that the underlying WM organization associated to cognitive functions were different in UHR individuals compared to healthy controls, and that specific regions were driving that difference.

Studies on patients with psychotic disorders have indicated that the relationship between WM-integrity and cognitive functions are altered by severe mental illness $(13,14)$. This structure-function relationship has motivated neurocognitive training programs as a means to induce neuroplastic effects on impaired cerebral networks in severe mental illness $(15,16)$. In a recent systematic review, we found evidence for controlled cognitive interventions, with a mean duration of 12 weeks, to be associated with WM-changes (17). One previous randomized clinical trial (RCT) on cognitive remediation in patients with schizophrenia reported regional increase in FA as an effect of treatment (18). However, there is a scarcity of studies on WM-neuroplasticity as a result of cognitive interventions in psychiatric patients, and to our knowledge none in UHR individuals. Cognitive remediation in UHR individuals is of particular interest, since these individuals may hold greater potential of brain plasticity, compared to patients with manifest psychotic disorders (19).

Cognitive remediation is a structured method of cognitive training, which is defined as "a behavioral training-based intervention that aims at improving cognitive processes (attention, memory, executive function, social cognition or metacognition) with the goal of durability and generalisation" (20). Thus, cognitive remediation targets selected cognitive domains and explicitly aims to improve social and neurocognitive functions in a manner that impacts real life functioning (21). There is meta-analytic evidence for the effectiveness of cognitive remediation in schizophrenia, with medium effect-sizes on global cognition and functional outcome (22), and the effects appear durable (20). Cognitive remediation is emphasized as an enhancing supplement to standard treatment, more effective when embedded in a broader psychiatric rehabilitation program (20). Studies are sparse in UHRpopulations. A recent systematic review (23), examining six studies on cognitive remediation with UHR individuals provided some preliminary evidence for the effectiveness of cognitive remediation on cognition [e.g improvement of verbal memory (ES 0.61) (24) and processing speed (ES 0.50) (25)], and aspects of functional outcome. However, methodological considerations were raised, such as low sample sizes and high attrition rates, precluding any solid conclusions to be drawn.

In this randomized clinical trial (Function and Overall Cognition in Ultra-high risk States, the FOCUS-trial), we aimed to evaluate the effect of comprehensive neurocognitive and social cognitive remediation in UHR individuals on cerebral WM organization, as assessed by fractional anisotropy. We hypothesized, that 20 weeks of 
cognitive remediation in addition to treatment as usual (TAU) would increase whole-brain fractional anisotropy in UHR individuals allocated to the intervention, when compared to UHR individuals allocated to TAU. Second, we investigated the effects of cognitive remediation on FA and additional WM-measures in $a$ priori ROIs. The regions were selected as the five regions our previous study had shown differentially associated to cognitive functions in UHR individuals. Next, we planned exploratory correlation-analyses between changes in WM-measures in the predefined regions, which showed significant interaction-effects on time ${ }^{\star}$ group and changes in clinical symptoms and cognitive functions. We expected correlations between changes in regional $\mathrm{WM}$ and changes in clinical symptoms/cognitive functions to be different, when comparing UHR individuals receiving cognitive remediation versus TAU. Finally, we explored a dose-response effect of cognitive remediation on WM.

\section{METHODS}

The current study is a sub study of a randomized, assessorblinded, parallel-group, superiority clinical trial comparing TAU plus 20 weeks of intensive cognitive remediation with TAU (the FOCUS-trial, registered at ClinicalTrials.gov NCT 02098408). The main trial protocol has been published (26), and the implications of the main results have been discussed in a separate paper (27). The study was carried out at the Mental Health Centre Copenhagen and Mental Health Centre Glostrup, University of Copenhagen, Denmark. Baseline data on the relationship between cognition and WM from the FOCUS-trial have been published elsewhere (10). Here, we analyzed longitudinal data from UHR individuals at two timepoints, pre-treatment (i.e. at baseline) and post-treatment (i.e. at 26weeks follow-up). All participants provided informed consent prior to inclusion into the study. The study protocol was approved by the Committee on Health Research Ethics of the Capital Region Denmark (study: H-6-2013-015) and the Danish Data Protection Agency (RHP-2014-009-02670).

\section{Procedures}

Following baseline assessments, participants were randomly assigned to TAU or TAU plus cognitive remediation for 20 weeks. The randomisation was centralised and computerised with a concealed randomisation sequence carried out by the Copenhagen Trial Unit (CTU). Randomisation was stratified by current use of antipsychotic medication (yes/no) and IQ score $(\leq 100 />100)$. Block size was blinded. Group allocation was concealed until the statistical analyses of the data had been completed. Investigators blinded to group allocation conducted assessments at 26-weeks (post-treatment) at a site remote from the intervention. Participants were instructed not to disclose their allocation prior to assessments. In case of failure of concealment, the assessment would be conducted by another research assessor. Assessors were psychologists and medical doctors with extensive training in the assessment instruments. Efficacy-analyses on WM were conducted by a blinded researcher.

\section{UHR Individuals}

UHR individuals were recruited from psychiatric in-and outpatient facilities in the catchment area of Copenhagen. Individuals were help-seeking individuals aged 18 to 40 years, who fulfilled one or more of the UHR criteria as assessed by the Comprehensive Assessment of At-Risk Mental State (CAARMS) (28): attenuated psychotic symptoms, and/or brief limited intermittent psychotic symptoms, and/or trait and vulnerability state along with a significant drop or sustained low functioning for the past year. Exclusion criteria were a history with a psychotic episode of more than one week of duration; psychiatric symptoms which were explained by a physical illness with psychotropic effect or acute intoxication; a diagnosis of a serious developmental disorder, or currently receiving methylphenidate.

\section{Assessments}

The CAARMS interview and The Structured Clinical Interview for DSM-IV Axis I Disorders (SCID-I) and part of the Structured Clinical Interview for DSM-IV Axis II Disorders (SCID-II) (29, 30) were used to diagnostically assess all UHR individuals. Level of general psychiatric symptoms was measured with the Brief Psychiatric Rating Scale Expanded Version (BPRS) (31), the level of negative symptoms was measured with the Scale for the Assessment of Negative Symptoms (SANS) (32), and the level of depressive symptoms was measured with the MontgomeyÅsberg Depression Rating Scale (MADRS) (33). Inter-rater reliability was assessed using intra-class correlations for the outcome measure SANS, BPRS, and MADRS in 12 interviews (ICC ratings from.96 to.99).

The third version of the Danish Weschler Adult Intelligence Scale (WAIS-III) (34) provided estimates of current level of intelligence. Cognitive functions were assessed using selected tests from the Brief Assessment of Cognition in Schizophrenia battery (BACS) (35): list-learning, digit sequencing, verbal fluency, and symbol coding, as well as tests from the Cambridge Neuropsychological Test Automated Battery (CANTAB) (36): Stockings of Cambridge (SOC Problems solved in minimum moves) and Intra-extradimensional set shifting test (IED Total errors adjusted, lower is better). For a detailed overview on cognitive domains and tests, see Supplementary Table S1.

\section{Image Acquisition and Processing}

MRI scans were acquired on a 3 Tesla scanner (Philips Healthcare, Best, the Netherlands). Diffusion-weighted images (DWIs) were acquired using single shot spin-echo echoplanar imaging (EPI) sequence with 30 noncollinear diffusion-weighted $[\mathrm{b}=1,000 \mathrm{~s} / \mathrm{mm}$ (2)] directions and one non-diffusion weighted $b=0 \mathrm{~s} / \mathrm{mm}$ (2). Two DWI scans were acquired, and the latter in an opposite phase encoding direction, enabling correction for susceptibility distortions (37). Details on image acquisition and processing are provided in Supplementary Text S2. Tools from the FSL software library v5.0.10 (38) and MRtrix3 (www.mrtrix.org) were used for image processing. Motion parameters were extracted to correct for head motion. Maps of fractional anisotropy (FA), axial diffusivity (AD), radial diffusivity $(\mathrm{RD})$ and mean diffusivity $(\mathrm{MD})$ were calculated. 
Tract-based spatial statistics (TBSS) (39) was used to create skeleton maps using a threshold of 0.2 . Using the JHU DTI-based white matter atlas labels (40), we computed the mean FA values in five WM-ROIs from skeletonized data. ROIs were selected $a$ priori and constituted the five regions where we in our previous cross-sectional study of baseline data had identified aberrant associations between WM and cognitive functions: fornix, medial lemniscus bilaterally, left superior cerebellar peduncle, and left uncinate fasciculus (10). MRI quality metrics were assessed by visual inspection and calculated from each subject using a quality assessment method described in Roalf et al. (41) (Table S3).

\section{Intervention}

The experimental intervention was delivered by a senior clinical psychologist with a specialization in psychotherapy and consisted of manualized neurocognitive and social cognitive remediation. The integrative approach targeting both neurocognitive and social cognitive deficits was based on the assumption, that neurocognitive and social cognitive remediation may work synergistically to increase the transfer of cognitive remediation gains to participants' real-world functioning (42).

Duration was $2 \mathrm{~h}$ once a week for a total of 20 weeks in a group-setting, combined with individual home-training. In addition to the group training, the participants received 12 individual sessions designed to maximize transfer of the effect of the cognitive training to the daily lives of the participants. The approach in individual sessions were cognitive behavioral therapy and targeted everyday challenges in relation to the UHR individual's specific cognitive deficits.

The neurocognitive remediation was performed using the Neuropsychological Educational Approach to Cognitive Remediation (NEAR) (43). Exercises from the web-based ScientificBrainTrainingpro.com and supplementary exercises from Brainhq.com were trained individually on computers for $1 \mathrm{~h}$ in the group-setting, followed by a group discussion aiming at relating the cognitive exercises to real world activities (bridging). UHR individuals were instructed to train the neurocognitive exercises at home to achieve the recommended dosage of $2 \mathrm{~h}$ per week of neurocognitive training ( $40 \mathrm{~h}$ in total). Homework was monitored in the training programs and motivation was addressed ongoing in the individual sessions, by sending text reminders and offering support from the group therapist. The therapist personalized the neurocognitive remediation based on the cognitive domains with the most severe impairments, as assessed by the UHR individuals baseline neurocognitive performance. The social cognitive training was performed using the Social Cognition and Interaction Training (SCIT) manual (44), which addresses several key social-cognitive domains. The therapist had attended a SCIT training course and received ad hoc supervision by Dr. David Roberts, first-author of the SCIT-manual (45). Adherence-rating to the treatment manual was conducted according to the SCIT Fidelity Scale (44) by an external rater.

Both the cognitive remediation group and the control group received TAU, which consisted of the treatment offered by the psychiatric services. TAU involved monitoring of medication, regular contact to primary practitioner delivering supportive counselling, and frequently a more regular psychotherapeutic intervention, such as social skills training, psycho-education, family-groups, supportive group-therapy, or individual therapeutic sessions with a psychologist, but not targeted cognitive remediation.

\section{Outcomes}

The primary outcome was whole brain fractional anisotropy (FA) as an effect of interaction between timepoint and treatment-group ('cognitive remediation versus TAU').

Secondary outcomes were measures of white matter: FA, axial diffusivity $(\mathrm{AD})$, radial diffusivity $(\mathrm{RD})$, and mean diffusivity (MD) in the five ROIs fornix, medial lemniscus bilaterally, left superior cerebellar peduncle, and left uncinate fasciculus, as an effect of interaction between timepoint and treatment-group ('cognitive remediation versus TAU').

Planned explorative tests were correlation analyses between WM-changes in the predefined ROIs, which showed significant between-group difference on secondary outcomes, and clinical and cognitive changes as compared between treatment-groups ('cognitive remediation versus TAU'). Moreover, as motivated by the potential dose-dependent effect of cognitive remediation on WM (17), we post-hoc tested a dose-response relationship between the proportion of cognitive remediation and change in whole-brain and regional WM.

\section{Statistical Analyses}

The main trial planned to enroll 126 UHR individuals. Powercalculation estimating sample-size was based on cognitive outcomes for the main trial (26).

Distribution of continuous data (clinical, cognitive and demographic data) was tested for normality, and group differences were tested using general linear modelling (GLM) co-varied for age and gender. Group differences in ordinal data (tobacco smoking, alcohol, drug use, and comorbidity) were tested using the Mann-Whitney $U$ test or Fisher's exact test. Nominal data were tested using Pearson's $\chi 2$ test. Group differences on baseline whole brain and regional FA were tested using univariate GLM and co-varied for age, gender, and relative and absolute head motion in the scanner.

Main analyses of treatment effect of cognitive remediation on primary and secondary outcomes were conducted using linear mixed models with repeated measurements and an unstructured covariance matrix as recommended for RCTs (46), assessing the interaction term between timepoint and group (cognitive remediation versus TAU). Covariates were age, gender, IQ, antipsychotic medication at baseline, and relative and absolute head motion in the scanner. Analyses were conducted according to the intention-to-treat principle, analyzing all participants, who completed MRI-scanning in the groups they were assigned to by randomization. Missing data were handled implicitly by the linear mixed modelling by full information maximum likelihood. Results were corrected for multiple comparisons using Bonferroni correction, and the significance threshold for the between-group analyses was set at $\mathrm{P} \leq 0.001(0.05 /((1$ whole brain +5 regions $) \times 4$ WM-measures $\mathrm{x} 2$ treatment-groups)) (47). 
Planned exploratory correlation analyses between changes in WM-measures on secondary outcomes and changes in clinical symptoms and cognitive functions, respectively, were conducted with bivariate correlation analyses using Pearson's $r$ or Spearmans rho as appropriate. In order to examine correlations specifically related to a potential treatment effect, testing was conducted on the predefined ROIs, which had shown an interaction-effect on time* group. Change-scores were calculated as: [numerical followup score - numerical baseline score]. Testing was performed with and without extreme outliers. If the main results remained unaffected, outliers were included in the analyses. Results were corrected for multiple comparisons using Bonferroni correction, and the significance threshold was set at $\mathrm{P} \leq 0.001(0.05 /(1 \mathrm{ROI} \times 6$ cognitive tests $\mathrm{x} 4 \mathrm{WM}$-measures $\mathrm{x} 2$ treatment-groups)).

Exploratory post-hoc analyses to test potential dose-response effect of cognitive remediation at the follow-up were conducted with linear mixed models with repeated measurements and an unstructured covariance matrix, with covarying for number of attended neurocognitive training-hours. All analyses were covaried for age, gender, IQ, antipsychotic medication, and head motion in scanner (relative/absolute).

All analyses were performed using IBM SPSS Statistics for Windows, Version 25.0, Armonk, NY, and the significance level was set to 0.05 (two-sided).

\section{RESULTS}

The main trial results have been described elsewhere (no effect of cognitive remediation on primary outcome of global neurocognition, or secondary outcomes of social- and occupational functioning, negative- or depressive symptoms) (27). In short, a total of 146 UHR individuals were assigned to either TAU or TAU + cognitive remediation (see Figure 1). Of these, 113 were MRI-scanned, and included in the current study.

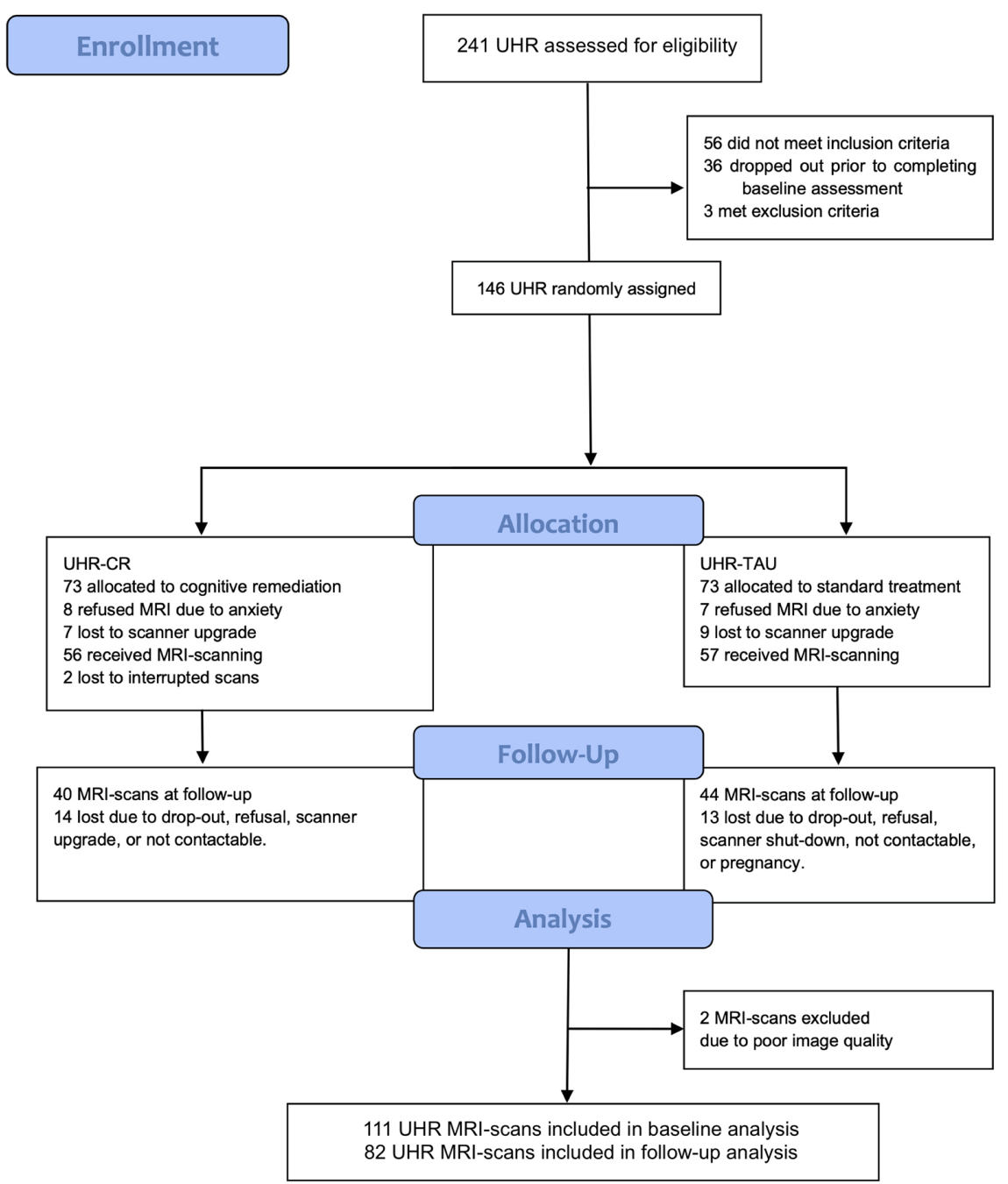

FIGURE 1 | Flowchart of the FOCUS trial. CR, cognitive remediation; MRI, magnetic resonance imaging; TAU, treatment as usual; UHR, ultra-high risk. 
Two MRI-scans were excluded due to poor image quality and extreme values. Thus, 111 UHR individuals were included in the analyses (Figure 1). Twenty UHR individuals discontinued the cognitive remediation intervention ( $18 \%$ attrition rate). Further nine UHR individuals did not complete follow-up scans due to practical issues (exceeded timeframe, scanner breakdown), resulting in a total of $82 \mathrm{UHR}$ individuals completing MRIscans at follow-up. The cognitive remediation group received significantly less TAU with a mean of $20 \mathrm{~h}$ within the 26 weeks intervention period compared to the TAU group (mean, $26 \mathrm{~h}$ ). The cognitive remediation group completed a mean of $12 \mathrm{~h}$ of neurocognitive training. Fidelity-rating to the SCIT treatment corresponded to an excellent adherence to the SCIT therapy manual, and inter-rater reliability on the clinical outcome measures showed excellent agreement (ICC ratings from 0.96 to 0.99). No adverse events were reported relating to the intervention.

Cognitive remediation and TAU-groups did not differ significantly on sociodemographic variables (Table 1), clinical symptoms or cognitive functions (Table 2). We found no significant difference on whole brain or regional FA between UHR individuals allocated to cognitive remediation or TAU on both timepoints (Figure 2). At 26-weeks follow-up, remission from UHR-status was similar in both cognitive remediation and TAU-groups ( $16 \%$ and $18 \%$ respectively); as well as conversionrates to psychosis ( $9 \%$ and $12 \%$ respectively) (Table 2 ).

The main mixed modelling analyses revealed no effect of the cognitive remediation on the primary outcome of whole-brain FA, with no significant interaction between timepoint ${ }^{\star}$ treatment $(\mathrm{P}=0.81$, Table 3$)$. Secondary outcome on regional WMmeasures revealed a nominal significant interaction between timepoint ${ }^{\star}$ treatment of $\mathrm{AD}$ in left medial lemniscus $(\mathrm{P}=0.016$, Table 3), which did not survive Bonferroni-correction for multiple comparisons.

Exploratory analyses of associations between change in WMmeasures in the region with significant between-group differences on secondary outcomes, and change in cognitive functions revealed a significant negative correlation between $\mathrm{WM}$ (AD increase) in left medial lemniscus, and changes in CANTAB IED (total errors adjusted decreased) ( $\mathrm{P}=0.001, \mathrm{CC}$ -0.54 ) for the cognitive remediation group, but not for TAU (Figure 3). No other correlations survived Bonferroni correction (See Supplementary Tables S4 and S5 for details).

In the exploratory analyses of dose-response effects in the cognitive remediation group, we found no effect when adding number of neurocognitive training hours as a covariate to WM-changes.

\section{DISCUSSION}

To our knowledge, this is the first randomized, clinical trial to evaluate the effect of cognitive remediation on white matter in UHR individuals. Contrary to our main hypothesis, we found no effect of cognitive remediation on the primary outcome of whole-brain FA. On our secondary outcome a nominal significant effect on one predefined white matter region (left
TABLE 1 | Sociodemographic data at baseline for UHR individuals.

\begin{tabular}{|c|c|c|}
\hline \multirow[t]{2}{*}{ Variable } & \multirow{2}{*}{$\begin{array}{c}\text { UHR-CR }(\mathrm{N}=54) \\
\text { Mean (S.D.)/ } \\
\text { No. (Percent) }\end{array}$} & \multirow{2}{*}{$\begin{array}{c}\text { UHR-TAU (N = 57) } \\
\text { Mean (S.D.)/ } \\
\text { No. (Percent) }\end{array}$} \\
\hline & & \\
\hline Age, mean (SD) & $23.5(4.8)$ & $24.1(3.6)$ \\
\hline \multicolumn{3}{|l|}{ Gender } \\
\hline Male & $26(48.1 \%)$ & $26(45.6 \%)$ \\
\hline Female & 28 (51.9\%) & $31(54.4 \%)$ \\
\hline \multicolumn{3}{|l|}{ Parental SES } \\
\hline Low & $6(11.1 \%)$ & $6(10.5 \%)$ \\
\hline Medium & 24 (44.4\%) & 17 (29.8\%) \\
\hline High & 24 (44.4\%) & 34 (59.6\%) \\
\hline \multicolumn{3}{|l|}{ Ethnicity } \\
\hline High-Income countries & 49 (92.5\%) & $48(88.9 \%)$ \\
\hline Low-Income countries & $4(7.4 \%)$ & $6(10.5 \%)$ \\
\hline BMI Mean (SD) & $22.6(3.4)$ & $24.1(5.4)$ \\
\hline \multicolumn{3}{|l|}{ Handedness } \\
\hline Right & 45 (83.3\%) & 52 (91.2\%) \\
\hline Left & $9(16.7 \%)$ & $5(8.8 \%)$ \\
\hline \multicolumn{3}{|l|}{ Function } \\
\hline SOFAS Mean (SD) & $55.6(11.9)$ & $54.7(10.1)$ \\
\hline $\begin{array}{l}\text { Activity-level (work and educational } \\
\text { hours per week) }\end{array}$ & $14.5(17.8)$ & $13.2(16.2)$ \\
\hline \multicolumn{3}{|l|}{ Alcohol consumption (last year) } \\
\hline Daily & $2(3.7 \%)$ & $1(1.8 \%)$ \\
\hline Weekly & $17(31.5 \%)$ & 18 (31.6\%) \\
\hline Monthly & 19 (35.2\%) & 21 (36.8\%) \\
\hline Once/twice & $6(11.1 \%)$ & $10(17.5 \%)$ \\
\hline Never & 10 (18.5\%) & 7 (12.3\%) \\
\hline \multicolumn{3}{|l|}{ Tobacco smoking (last year) } \\
\hline Daily & 25 (46.3\%) & $22(38.6 \%)$ \\
\hline Weekly & $3(5.6 \%)$ & $3(5.3 \%)$ \\
\hline Monthly & $1(1.9 \%)$ & $3(5.3 \%)$ \\
\hline Once/twice & $4(7.4 \%)$ & $2(3.5 \%)$ \\
\hline Never & 21 (38.9\%) & 27 (47.4\%) \\
\hline \multicolumn{3}{|l|}{ Cannabis smoking (last year) } \\
\hline Daily & $3(5.6 \%)$ & $0(0.0 \%)$ \\
\hline Weekly & $1(1.9 \%)$ & $4(7.1 \%)$ \\
\hline Monthly & $6(11.1 \%)$ & $1(1.8 \%)$ \\
\hline Once/twice & $9(16.7 \%)$ & $8(14.3 \%)$ \\
\hline Never & $35(64.8 \%)$ & $43(76.8 \%)$ \\
\hline
\end{tabular}

$B M I$, body-mass index; $C R$, cognitive remediation; No., number; $S D$, standard deviation; SES, socio-economic status; SOFAS, social and occupational function assessment scale; TAU, treatment as usual; UHR, ultra-high risk.

medial lemniscus) did not survive adjustment for multiple comparisons. The lack of global and regional WM-changes as a result of cognitive remediation we found are in line with the results from the main trial (27), which did not result in improvements in global measures of cognition, function, or clinical symptoms, but marginal effects on exploratory outcomes (i.e. emotion recognition). Nor did our doseresponse analyses on completed hours of neurocognitive training in the cognitive remediation group support an effect on FA in whole brain or ROIs.

The result could, beyond the conclusion of no effect of cognitive remediation on $\mathrm{WM}$, be explained by the low number of neurocognitive training sessions. The average of $12 \mathrm{~h}$ of neurocognitive training is considerably lower than the recommended dosage (25-30 h) assumed necessary for driving cognitive improvements (48). A controlled, randomized trial on 
TABLE 2 | Clinical and cognitive data at baseline and follow-up for UHR individuals.

\begin{tabular}{|c|c|c|c|c|}
\hline \multirow[t]{2}{*}{ Variable } & \multicolumn{2}{|c|}{$\begin{array}{c}\text { UHR-CR } \\
\text { Mean (S.D.)/No. (Percent) }\end{array}$} & \multicolumn{2}{|c|}{$\begin{array}{c}\text { UHR-TAU } \\
\text { Mean (S.D.)/No. (Percent) }\end{array}$} \\
\hline & Baseline ( $N=54)$ & Follow-Up $(N=43)$ & Baseline $(\mathrm{N}=57$ ) & Follow-up ( $N=48)$ \\
\hline \multicolumn{5}{|l|}{ Medication } \\
\hline Antipsychotic-naive & $34(64.2 \%)$ & $16(37.2 \%)$ & 29 (50.9\%) & $12(25.0 \%)$ \\
\hline Current $^{\dagger}$ antipsychotics ${ }^{\star}$ & $14(26.4 \%)$ & 24 (55.8\%) & $21(36.8 \%)$ & $33(68.8 \%)$ \\
\hline Current $^{\dagger}$ antidepressants & $13(24.5 \%)$ & $12(28.6 \%)$ & $16(28.1 \%)$ & $11(22.9 \%)$ \\
\hline Current $^{\dagger}$ mood stabilizers & $2(3.8 \%)$ & $5(11.6 \%)$ & $4(7.0 \%)$ & $4(8.3 \%)$ \\
\hline Current $^{\dagger}$ benzodiazepines & $1(1.9 \%)$ & $6(14.0 \%)$ & 7 (12.3\%) & $4(8.3 \%)$ \\
\hline Diagnose of current ${ }^{\dagger}$ abuse & $0(0.0 \%)$ & $0(0.0 \%)$ & $1(1.9 \%)$ & $0(0.0 \%)$ \\
\hline Diagnose of current ${ }^{\dagger}$ dependency & $0(0.0 \%)$ & $0(0.0 \%)$ & $1(1.9 \%)$ & $0(0.0 \%)$ \\
\hline \multicolumn{5}{|l|}{ Clinical outcomes } \\
\hline CAARMS composite & $51.9(11.9)$ & $38.1(16.7)$ & 49.7 (16.5) & $33.2(15.3)$ \\
\hline SANS & $1.6(0.7)$ & $1.4(0.9)$ & $1.5(0.8)$ & $1.1(1.6)$ \\
\hline BPRS & $42.9(7.6)$ & $37.8(12.6)$ & $41.0(10.7)$ & $38.0(10.8)$ \\
\hline MADRS & $16.3(7.4)$ & $12.0(7.5)$ & $15.0(7.0)$ & $13.1(7.2)$ \\
\hline Remission from UHR-status & & $7(16.3 \%)$ & & $8(17.8 \%)$ \\
\hline Conversion to psychosis & & $5(9.3 \%)$ & & $7(12.3 \%)$ \\
\hline Drop-out & & $11(20.4 \%)$ & & $9(15.8 \%)$ \\
\hline \multicolumn{5}{|l|}{ Cognitive functions } \\
\hline IQ & $102.8(12.7)$ & & $104.5(12.4)$ & \\
\hline \multicolumn{5}{|l|}{ BACS } \\
\hline List-Learning & $51.7(8.9)$ & $53.2(8.8)$ & $50.4(7.8)$ & $53.1(9.4)$ \\
\hline Digit sequencing & $21.0(3.9)$ & $21.1(4.1)$ & $20.0(4.4)$ & $21.1(4.2)$ \\
\hline Fluency & $58.2(15.2)$ & $59.2(18.6)$ & $57.4(11.7)$ & $59.9(12.3)$ \\
\hline Symbol coding & $57.3(11.3)$ & $62.2(11.7)$ & $59.0(11.5)$ & $62.3(12.7)$ \\
\hline \multicolumn{5}{|l|}{ CANTAB } \\
\hline SOC & $9.8(1.7)$ & $10.8(2.1)$ & $10.1(1.8)$ & $10.3(1.6)$ \\
\hline IED & $18.6(16.0)$ & $20.1(34.8)$ & $19.2(16.0)$ & $11.4(10.0)$ \\
\hline
\end{tabular}

The clinical characteristics and cognitive functions for UHR individuals at baseline and follow-up are shown.

^Atypical antipsychotics in low dose: aripiprazole, amilsulpride, olanzapine, paliperidone, quetiapine, risperidone.

+ Current, the last month.

APS, attenuated psychotic symptoms; BACS, brief assessment of cognition in schizophrenia battery; BLIPS, brief limited intermittent psychotic symptoms; CAARMS, comprehensive assessment of at-risk mental state; CANTAB< Cambridge neuropsychological test automated battery; CR, cognitive remediation; IED, intra-extradimensional set shifting test, total errors, adjusted; IQ, intelligence quotient; N, count; SD, standard deviation; SOC, stockings of Cambridge, Problems solved in minimum moves; UHR, ultra-high risk.

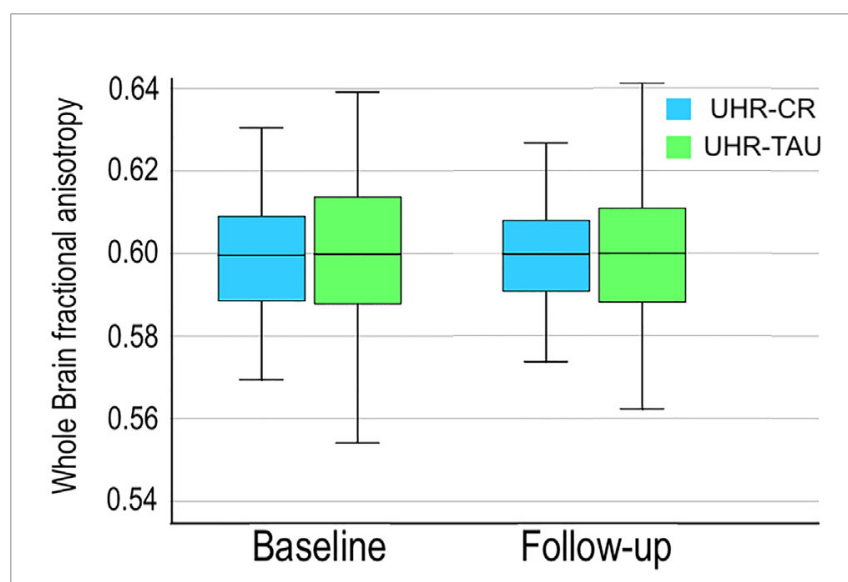

FIGURE 2 | Boxplots illustrating the development of mean whole brain fractional anisotropy at baseline and 26 weeks follow-up for UHR individuals allocated to cognitive remediation versus treatment as usual are displayed. Error bars indicate the $95 \%$ confidence intervals. Note that the $y$-axis has been altered to enhance visual display. CR, cognitive remediation; TAU, treatment as usual; UHR, ultra-high risk. patients with schizophrenia reported the neuroplastic effects of cognitive remediation (increased FA in corpus callosum), which was correlated to cognitive executive functions (18). However, this intervention group was exposed to 40 1-h sessions of neurocognitive training with a higher frequency, which is a considerably larger dose than the UHR individuals in the current trial. The question of sufficient dosage to induce consistent WMchanges might be particularly important when evaluating neuroplasticity-based treatment. A multistage model for associations between learning and cerebral changes would explain how rapid improvements in learning and behavior may be linked to transient and elastic WM-changes (49), which tend to return to baseline at subsequent follow-ups (50). Consolidation and automatization of training and learning, which would be accompanied by more robust neuroplastic reorganization, would appear within a longer time-frame (51). Indeed, several studies have demonstrated a sustained effect on WM-measures, suggesting a temporal dynamic in progressive WM-reorganization associated with increased skills and new learning (52-54). However, the underlying biological mechanisms involved in these timedependent variations are not straightforward, as multiple cellular 
TABLE 3 | White matter characteristics at baseline and follow-up in individuals at ultra-high risk for psychosis.

\begin{tabular}{|c|c|c|c|c|c|c|c|c|c|c|}
\hline & & & \multicolumn{2}{|c|}{$\begin{array}{c}\text { FA } \\
\text { Mean (SD) }\end{array}$} & \multicolumn{2}{|c|}{$\begin{array}{c}\text { AD } \\
\text { Mean (SD) } \\
\times 10^{-2}\end{array}$} & \multicolumn{2}{|c|}{$\begin{array}{c}\text { RD } \\
\text { Mean (SD) } \\
\times 10^{-2}\end{array}$} & \multicolumn{2}{|c|}{$\begin{array}{c}\text { MD } \\
\text { Mean (SD) } \\
\times 10^{-2}\end{array}$} \\
\hline & & & CR & TAU & CR & TAU & CR & TAU & CR & TAU \\
\hline \multirow[t]{4}{*}{ Primary outcomes } & Whole Brain & BL & $\begin{array}{l}0.5782 \\
(0.0139)\end{array}$ & $\begin{array}{l}0.5805 \\
(0.0167)\end{array}$ & $\begin{array}{l}0.1360 \\
(0.0024)\end{array}$ & $\begin{array}{l}0.1361 \\
(0.0033)\end{array}$ & $\begin{array}{l}0.0462 \\
(0.0019)\end{array}$ & $\begin{array}{l}0.0459 \\
(0.0025)\end{array}$ & $\begin{array}{l}0.0761 \\
(0.0018)\end{array}$ & $\begin{array}{l}0.0759 \\
(0.0024)\end{array}$ \\
\hline & & FU & $\begin{array}{l}0.5791 \\
(0.0160)\end{array}$ & $\begin{array}{l}0.5814 \\
(0.0160)\end{array}$ & $\begin{array}{l}0.1354 \\
(0.0021)\end{array}$ & $\begin{array}{l}0.1357 \\
(0.0028)\end{array}$ & $\begin{array}{l}0.0459 \\
(0.0022)\end{array}$ & $\begin{array}{l}0.0456 \\
(0.0023)\end{array}$ & $\begin{array}{l}0.0757 \\
(0.0019)\end{array}$ & $\begin{array}{l}0.0756 \\
(0.0021)\end{array}$ \\
\hline & & Interaction Time*Group & \multicolumn{2}{|c|}{$P=0.81$} & \multicolumn{2}{|c|}{$P=0.85$} & \multicolumn{2}{|c|}{$P=0.28$} & \multicolumn{2}{|c|}{$P=0.92$} \\
\hline & & & \multicolumn{2}{|c|}{$F=0.06$} & \multicolumn{2}{|c|}{$F=0.04$} & \multicolumn{2}{|c|}{$F=1.18$} & \multicolumn{2}{|c|}{$F=0.01$} \\
\hline \multirow[t]{29}{*}{ Secondary outcomes } & Fornix & BL & $\begin{array}{l}0.5656 \\
(0.0745)\end{array}$ & $\begin{array}{l}0.5546 \\
(0.0625)\end{array}$ & $\begin{array}{l}0.1985 \\
(0.0196)\end{array}$ & $\begin{array}{l}0.2033 \\
(0.0146)\end{array}$ & $\begin{array}{l}0.0797 \\
(0.0025)\end{array}$ & $\begin{array}{l}0.0829 \\
(0.0018)\end{array}$ & $\begin{array}{l}0.1193 \\
(0.0229)\end{array}$ & $\begin{array}{l}0.1231 \\
(0.0166)\end{array}$ \\
\hline & & FU & 0.5594 & 0.5543 & 0.1999 & 0.2022 & 0.0814 & 0.0829 & 0.1209 & 0.1227 \\
\hline & & & $(0.0837)$ & $(0.0649)$ & $(0.0211)$ & $(0.0166)$ & $(0.0029)$ & $(0.0019)$ & $(0.0258)$ & $(0.0177)$ \\
\hline & & Interaction Time*Group & \multicolumn{2}{|c|}{$P=0.79$} & \multicolumn{2}{|c|}{$P=0.64$} & \multicolumn{2}{|c|}{$P=0.62$} & \multicolumn{2}{|c|}{$P=0.59$} \\
\hline & & & \multicolumn{2}{|c|}{$F=0.23$} & \multicolumn{2}{|c|}{$F=0.44$} & \multicolumn{2}{|c|}{$F=0.48$} & \multicolumn{2}{|c|}{$F=0.53$} \\
\hline & Right medial lemniscus & BL & 0.6313 & 0.6336 & 0.1399 & 0.1395 & 0.0440 & 0.0435 & 0.0760 & 0.0755 \\
\hline & & & (0.0384) & $(0.0249)$ & (0.0068) & (0.0062) & $(0.0048)$ & $(0.0031)$ & $(0.0045)$ & (0.0033) \\
\hline & & FU & 0.6302 & 0.6411 & 0.1386 & 0.1389 & 0.0437 & 0.0424 & 0.0753 & 0.0746 \\
\hline & & & (0.0346) & $(0.0265)$ & $(0.0059)$ & $(0.0065)$ & (0.0039) & $(0.0031)$ & $(0.0035)$ & $(0.0035)$ \\
\hline & & Interaction Time*Group & \multicolumn{2}{|c|}{$P=0.76$} & \multicolumn{2}{|c|}{$P=0.33$} & \multicolumn{2}{|c|}{$P=0.69$} & \multicolumn{2}{|c|}{$P=0.54$} \\
\hline & & & $\mathrm{F}=\mathrm{C}$ & .28 & $\mathrm{~F}=$ & .11 & $F=($ & .38 & $\mathrm{~F}=\mathrm{C}$ & 62 \\
\hline & Left medial lemniscus & BL & 0.6179 & 0.6253 & 0.1396 & 0.1387 & 0.0456 & 0.0443 & 0.0769 & 0.0758 \\
\hline & & & (0.0366) & $(0.0334)$ & $(0.0064)$ & $(0.0067)$ & $(0.0045)$ & $(0.0040)$ & $(0.0041)$ & $(0.0040)$ \\
\hline & & FU & 0.6266 & 0.6341 & 0.1399 & 0.1367 & 0.0447 & 0.0428 & 0.0764 & 0.0741 \\
\hline & & & (0.0318) & $(0.0327)$ & $(0.0053)$ & $(0.0072)$ & $(0.0041)$ & (0.0042) & $(0.0036)$ & $(0.0044)$ \\
\hline & & Interaction Time*Group & $P=($ & .37 & $\mathbf{P}=$ & .02 & $P=$ & .41 & $P=0$ & .14 \\
\hline & & & $\mathrm{F}=\mathrm{C}$ & .99 & $\mathrm{~F}=$ & .74 & $F=($ & .90 & $F=2$ & .03 \\
\hline & Left superior cerebellar & BL & 0.6422 & 0.6492 & 0.1618 & 0.1613 & 0.0498 & 0.0488 & 0.0872 & 0.0863 \\
\hline & peduncle & & (0.0319) & $(0.0373)$ & $(0.0071)$ & $(0.0074)$ & $(0.0046)$ & (0.0059) & (0.0043) & $(0.0054)$ \\
\hline & & FU & 0.6403 & 0.6472 & 0.1602 & 0.1593 & 0.0495 & 0.0486 & 0.0864 & 0.0855 \\
\hline & & & $(0.0311)$ & $(0.0342)$ & $(0.0058)$ & $(0.0075)$ & (0.0049) & $(0.0055)$ & $(0.0044)$ & $(0.0055)$ \\
\hline & & Interaction Time*Group & $P=$ & .40 & $\mathrm{P}=$ & .18 & $P=$ & .17 & $P=C$ & .12 \\
\hline & & & $\mathrm{F}=\mathrm{C}$ & .92 & $\mathrm{~F}=$ & .74 & $F=$ & .82 & $F=2$ & .20 \\
\hline & Left uncinate fasciculus & BL & 0.5034 & 0.5027 & 0.1277 & 0.1279 & 0.0541 & 0.0543 & 0.0786 & 0.0788 \\
\hline & & & $(0.0412)$ & $(0.0393)$ & $(0.0065)$ & $(0.0066)$ & $(0.0037)$ & $(0.0031)$ & $(0.0029)$ & (0.0026) \\
\hline & & FU & 0.5017 & 0.5009 & 0.1272 & 0.1277 & 0.0541 & 0.0544 & 0.0785 & 0.0788 \\
\hline & & & $(0.0431)$ & (0.0369) & $(0.0054)$ & $(0.0067)$ & $(0.0042)$ & $(0.0029)$ & $(0.0030)$ & (0.0026) \\
\hline & & Interaction Time*Group & $P=($ & .77 & $\mathrm{P}=$ & .94 & $\mathrm{P}=$ & .92 & $P=C$ & .74 \\
\hline & & & $\mathrm{F}=\mathrm{C}$ & 27 & $\mathrm{~F}=$ & .06 & $\mathrm{~F}=\mathrm{C}$ & .08 & $\mathrm{~F}=\mathrm{C}$ & \\
\hline
\end{tabular}

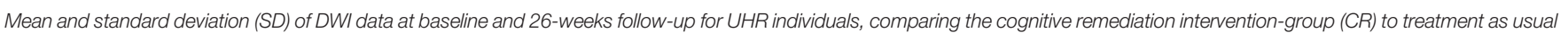

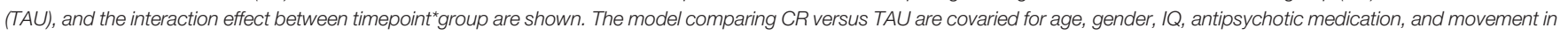
scanner. Group*Time interaction significant before correction for multiple comparisons are marked in bold.

$A D$, axial diffusivity; $C R$, cognitive remediation; DWI, diffusion weighted imaging; FA, fractional anisotropy; $M D$, mean diffusivity; RD, radial diffusivity; SD, standard deviation; BL, Baseline;

FU, Follow-up; TAU, treatment as usual; UHR, ultra-high risk.

processes may be associated with WM-measures such as FA. Various candidate mechanisms of activity-dependent WMreorganization has been suggested, such as long-term potentiation (55), growth of astrocytes and oligodendrocyte precursor cells (56), myelination (57), and reorganization of axons and fiber-bundles (58). Our results stress the perspective of investigating duration and intensity as prerequisites of neuroplasticity-based interventions embedded in a broader clinical setting. Moreover, future research specifying the potential active treatment ingredients beyond duration and intensity, such as choice of remediation strategies (i.e. integrative versus simple programs, or compensatory versus restorative remediation approaches) may support the clinical tailoring of treatment strategies. The clinical perspectives includes enhancement of motivation and attendance $(21,59)$, as well as the question of whether this comprehensive treatment may only be viable for the subgroup of UHR individuals who can be engaged sufficiently to practice the skills in the recommended dosage.

Exploratory analyses revealed mental flexibility to be correlated with a change of left medial lemniscus $\mathrm{AD}$ in the cognitive remediation group, but not in the TAU-group. Although our observation is marginal and should be interpreted very cautiously, medial lemniscus is part of the cerebello-thalamocortical connections [CTC (60)], which previously has been associated with symptom course in UHR individuals $(61,62)$. Interestingly, our observation would be consistent with a recent study in patients with schizophrenia, reporting that WM organization in medial lemniscus predicted training induced improvements in executive functioning, and suggested that preserved WM organization in 


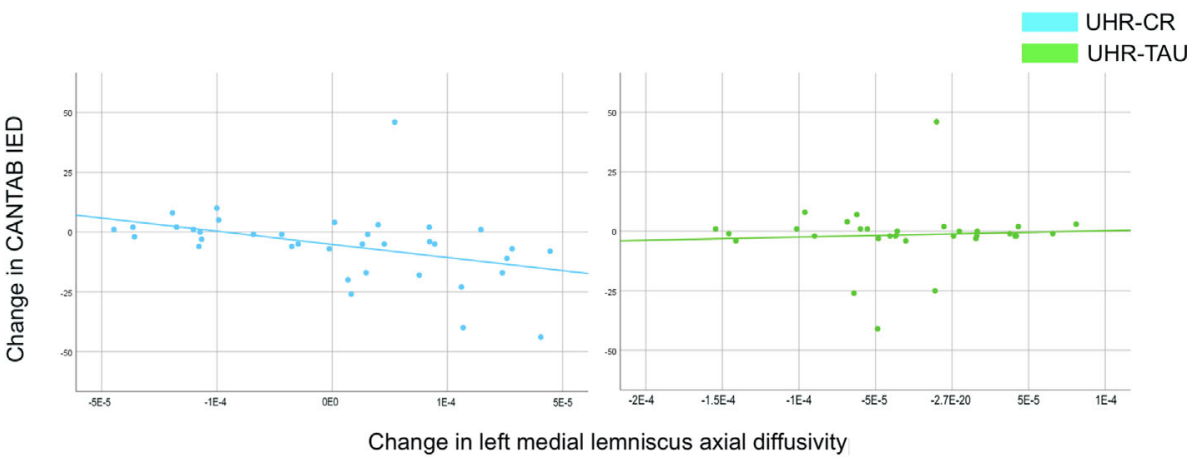

FIGURE 3 | The scatterplots illustrate the exploratory correlations between changes in left medial lemniscus axial diffusivity and changes in mental flexibility (CANTAB IED total errors adjusted) in UHR individuals allocated to cognitive remediation versus treatment as usual are displayed. Error-bars indicate the 95\% confidence intervals. Note that the $y$-axis has been altered to enhance visual display. The correlations have been tested with and without outliers, which were included due to no effect on the result. Numerical results are reported in detail in Supplementary Table S4. CANTAB, Cambridge neuropsychological test automated battery; CR, cognitive remediation; IED, intra-extra dimensional set-shifting; TAU, treatment as usual; UHR, ultra-high risk.

CTC-connections may be an important determinant for training induced neurocognitive plasticity (63). We speculate whether this correlation may indicate a subtle protective effect of cognitive remediation on loss of WM-integrity. The protective approach of intact cognitive functions have been discussed as an alternative to focusing on restoration of impaired functions by cognitive remediation (64). Our observations lend support to further investigations of strategies for cognitive remediation, i.e. specifically aimed at preserving targeted cognitive functions while potentially preventing WM-deterioration in early psychosis, versus the integrative program combining various remediation strategies as applied in the current trial.

A strength of this study is that it was undertaken as part of a high quality randomized, assessor-blinded clinical trial. Limiting the study is the moderate attrition-rate, along with the low number of mean neurocognitive training hours, which impede reaching firm conclusions about the effect of cognitive remediation delivered per the trial-protocol. Furthermore, sample-size was calculated on different outcomes from the main trial, which is a weakness in our study-design. However, this study presents a large sample-size compared to previous studies examining neuroplastic effects of cognitive interventions.

\section{CONCLUSION}

Cognitive remediation comprising $12 \mathrm{~h}$ of neurocognitive training on average did not improve global or regional WM organization in UHR individuals. Further investigations of the duration and intensity of neuroplasticity-based cognitive training as necessary prerequisites of WM-changes are warranted.

\section{DATA AVAILABILITY STATEMENT}

The data sets presented in this article are not readily available because: Not allowed by the Danish Data Protection Agency.
Requests to access the datasets should be directed to TK, tina.dam.kristensen@regionh.dk.

\section{ETHICS STATEMENT}

The studies involving human participants were reviewed and approved by The study protocol was approved by the Committee on Health Research Ethics of the Capital Region Denmark (study: H-6-2013-015) and the Danish Data Protection Agency (RHP-2014-009-02670). The patients/participants provided their written informed consent to participate in this study.

\section{AUTHOR CONTRIBUTIONS}

$\mathrm{MN}, \mathrm{LG}$, and BF conceived and designed the trial protocol. MN, $\mathrm{BG}, \mathrm{LG}, \mathrm{CH}, \mathrm{TK}$, and $\mathrm{CW}$ raised the funding. JJ and $\mathrm{BF}$ supervised the data collection. BE, RM, and CP supervised the study. JR processed MRI-data. TK conducted the analyses supervised by $\mathrm{CH}$. TK wrote the initial draft. All authors contributed to the article and approved the submitted version.

\section{FUNDING}

The study was funded by The Danish Council for Independent Research (DFF-4004-00314); TrygFoundation (108119); the Mental Health Services in the Capital Region of Denmark; the research fund of the Capital Region of Denmark; the Lundbeck Foundation Center for Clinical Intervention and Neuropsychiatric Schizophrenia Research, CINS (R155-2013-16337). CP was supported by a NHMRC Senior Principal Research Fellowship (1105825) and by a grant from Lundbeck Foundation (R2462016-3237). 


\section{ACKNOWLEDGMENTS}

We thank the participants and clinical staff for their participation. In particular, we are grateful that Lise Mariegaard as the senior psychologist delivered the FOCUS-intervention with excellence.

\section{REFERENCES}

1. Fusar-Poli P, Bonoldi I, Yung AR, Borgwardt S, Kempton MJ, Valmaggia L, et al. Predicting Psychosis. Arch Gen Psychiatry (2012) 69(3):220-9. doi: 10.1001/archgenpsychiatry.2011.1472

2. Bora E, Lin A, Wood SJ, Yung AR, Mcgorry PD, Pantelis C. Cognitive deficits in youth with familial and clinical high risk to psychosis: A systematic review and meta-analysis. Acta Psychiatr Scand (2014) 130(1):1-15. doi: 10.1111/ acps. 12261

3. Fusar-Poli P, Deste G, Smieskova R, Barlati S, Yung AR, Howes O, et al. Cognitive Functioning in Prodromal Psychosis. Arch Gen Psychiatry (2012) 69(6):562-71. doi: 10.1001/archgenpsychiatry.2011.1592

4. De Herdt A, Wampers M, Vancampfort D, De Hert M, Vanhees L, Demunter $\mathrm{H}$, et al. Neurocognition in clinical high risk young adults who did or did not convert to a first schizophrenic psychosis: A meta-analysis. Schizophr Res (2013) 149(1-3):48-55. doi: 10.1016/j.schres.2013.06.017

5. Mam-lam-Fook C, Danset-Alexandre C, Pedron L, Amado I, Gaillard R, Krebs M-O. Neuropsychology of subjects with ultra-high risk (UHR) of psychosis: A critical analysis of the literature. Encephale (2017) 43(3):241-53. doi: 10.1016/j.encep.2017.02.001

6. de Paula ALD, Hallak JEC, Maia-de-Oliveira JP, Bressan RA, Machado-deSousa JP. Cognition in at-risk mental states for psychosis. Neurosci Biobehav Rev (2015) 57:199-208. doi: 10.1016/j.neubiorev.2015.09.006

7. Vijayakumar N, Bartholomeusz C, Whitford T, Hermens DF, Nelson B, Rice $\mathrm{S}$, et al. White matter integrity in individuals at ultra-high risk for psychosis: a systematic review and discussion of the role of polyunsaturated fatty acids. BMC Psychiatry (2016) 16(1):1. doi: 10.1186/s12888-016-0932-4

8. Le Bihan D, Iima M. Diffusion magnetic resonance imaging: What water tells us about biological tissues. PloS Biol (2015) 13(7):1-13. doi: 10.1371/ journal.pbio.1002203

9. Alexander AL, Lee JE, Lazar M, Field AS. Diffusion tensor imaging of the brain. Neurotherapeutics (2007) 4(3):316-29. doi: 10.1016/j.nurt.2007.05.011

10. Kristensen TD, Mandl RCW, Raghava JM, Jessen K, Jepsen JRM, Fagerlund B, et al. Widespread higher fractional anisotropy associates to better cognitive functions in individuals at ultra-high risk for psychosis. Hum Brain Mapp (2019) 40(18):5185-201. doi: 10.1002/hbm.24765

11. Krakauer K, Ebdrup BH, Glenthoj BY, Raghava JM, Nordholm D, Randers L, et al. Patterns of white matter microstructure in individuals at ultra-high-risk for psychosis: Associations to level of functioning and clinical symptoms. Psychol Med (2017) 47(15):2689-707. doi: 10.1017/S0033291717001210

12. Peters BD, Dingemans PM, Dekker N, Blaas J, Akkerman E, van Amelsvoort TA, et al. White matter connectivity and psychosis in ultra-high-risk subjects: A diffusion tensor fiber tracking study. Psychiatry Res - Neuroimag (2010) 181 (1):44-50. doi: 10.1016/j.pscychresns.2009.10.008

13. Nazeri A, Chakravarty MM, Felsky D, Lobaugh NJ, Rajji TK, Mulsant BH, et al. Alterations of superficial white matter in schizophrenia and relationship to cognitive performance. Neuropsychopharmacology (2013) 38(10):1954-62. doi: $10.1038 /$ npp.2013.93

14. Penades R, Vallespi LG, Franck N, Dekerle M. Neuroimaging Studies of Cognitive Function in Schizophrenia Chapter. Adv Exp Med Biol (2019) 1118 (January):v-vi. doi: 10.1007/978-3-030-05542-4_6

15. Fisher M, Loewy R, Hardy K, Schlosser D, Vinogradov S. Cognitive Interventions Targeting Brain Plasticity in the Prodromal and Early Phases of Schizophrenia. Annu Rev Clin Psychol (2013) 9:435-63. doi: 10.1146/ annurev-clinpsy-032511-143134

16. Vinogradov S, Fisher M, de Villers-Sidani E. Cognitive training for impaired neural systems in neuropsychiatric illness. Neuropsychopharmacology (2012) 37(1):43-76. doi: 10.1038/npp.2011.251

17. Kristensen TD, Mandl RCW, Jepsen JRMM, Rostrup E, Glenthøj LB, Nordentoft M, et al. Non-pharmacological modulation of cerebral white

\section{SUPPLEMENTARY MATERIAL}

The Supplementary Material for this article can be found online at: https://www.frontiersin.org/articles/10.3389/fpsyt.2020. 00873/full\#supplementary-material

matter organization: A systematic review of non-psychiatric and psychiatric studies. Neurosci Biobehav Rev (2018) 88(August 2017):84-97. doi: 10.1016/ j.neubiorev.2018.03.013

18. Penadés R, Pujol N, Catalán R, Massana G, Rametti G, Garcıa-Rizo C, et al. Brain effects of cognitive remediation therapy in schizophrenia: A structural and functional neuroimaging study. Biol Psychiatry (2013) 73(10):1015-23. doi: 10.1016/j.biopsych.2013.01.017

19. Keshavan MS, Hogarty GE. Brain maturational processes and delayed onset in schizophrenia. Dev Psychopathol (1999) 11(3):525-43. doi: 10.1017/ S0954579499002199

20. Wykes T, Huddy V, Cellard C, McGurk SR, Czobor P. A meta-analysis of cognitive remediation for schizophrenia: Methodology and effect sizes. Am J Psychiatry (2011) 168(5):472-85. doi: 10.1176/appi.ajp.2010.10060855

21. Mogami T. Cognitive remediation for schizophrenia with focus on NEAR. Front Psychiatry (2018) 8:304(JAN). doi: 10.3389/fpsyt.2017.00304

22. McGurk SR, Twamley EW, Sitzer DI, J GM, Mueser KT. A Meta-Analysis of Cognitive Remediation in Schizophrenia. Am J Psychiatry (2007) 164 (12):1791-802. doi: 10.1176/appi.ajp.2007.07060906.A

23. Glenthøj LB, Hjorthøj C, Kristensen TD, Davidson CA, Nordentoft M. The effect of cognitive remediation in individuals at ultra-high risk for psychosis: a systematic review. NPJ Schizophr (2017) 3(1):20. doi: 10.1038/s41537-0170021-9

24. Loewy R, Fisher M, Schlosser DA, Biagianti B, Stuart B, Mathalon DH, et al. Intensive Auditory Cognitive Training Improves Verbal Memory in Adolescents and Young Adults at Clinical High Risk for Psychosis. Schizophr Bull (2016) 42 Suppl 1:S118-26. doi: 10.1093/schbul/sbw009. sbw009.

25. Choi J, Corcoran CM, Fiszdon JM, Stevens M, Javitt DC, Deasy M, et al. Pupillometer-Based Neurofeedback Cognitive Training to Improve Processing Speed and Social Functioning in Individuals at Clinical High Risk for Psychosis. Psychiatr Rehabil J (2016) 40(1):33-42. doi: 10.1037/ prj0000217

26. Glenthøj LB, Fagerlund B, Randers L, Hjorthøj CR, Wenneberg C, Krakauer $\mathrm{K}$, et al. The FOCUS trial: cognitive remediation plus standard treatment versus standard treatment for patients at ultra-high risk for psychosis: study protocol for a randomised controlled trial. Trials (2015) 16(1):1-10. doi: 10.1186/s13063-014-0542-8

27. Glenthøj LB, Mariegaard LS, Fagerlund B, Jepsen JRM, Kristensen TD, Wenneberg $\mathrm{C}$, et al. Cognitive remediation plus standard treatment versus standard treatment alone for individuals at ultra-high risk of developing psychosis: results of the FOCUS randomised clinical trial. Schizophrenia Res (2020) 19:2(September):54-5. doi: 10.1002/wps.20760

28. Yung AR, Yuen HP, McGorry PD, Phillips LJ, Kelly D, Dell'Olio M, et al. Mapping the onset of psychosis: The Comprehensive Assessment of At-Risk Mental States. Aust N Z J Psychiatry (2005) 39(11-12):964-71. doi: 10.1111/ j.1440-1614.2005.01714.x

29. First MB, Gibbon M. The Structured Clinical Interview for DSM-IV Axis I Disorders (SCID-I) and the Structured Clinical Interview for DSM-IV Axis II Disorders (SCID-II). In: Comprehensive Handbook of Psychological Assessment, Vol. 2: Personality Assessment. Hoboken, NJ, US: John Wiley \& Sons Inc (2004). p. 134-43.

30. Spitzer RL, Williams JBW, Gibbon M, First MB. User"s Guide for the Structured Clinical Interview for DSM-III-R: SCID. Arlington, VA, US: American Psychiatric Association (1990).

31. Ventura J, Nuechterlein KH, Subotnik KL, Gutkind D, Gilbert E a. Symptom dimensions in recent-onset schizophrenia and mania: A principal components analysis of the 24-item Brief Psychiatric Rating Scale. Psychiatry Res (2000) 97(2-3):129-35. doi: 10.1016/S0165-1781(00)00228-6

32. Andreasen NC. Scale for the Assessment of Negative Symptoms. Iowa City, Iowa: Dept. of Psychiatry, College of Medicine, the University of Iowa (1984). 
33. Montgomery S a., Asberg M. A new depression scale designed to be sensitive to change. Br J Psychiatry (1979) 134(4):382-9. doi: 10.1192/bjp.134.4.382

34. Wechsler D. Wais-3 Administration and Scoring Manual. San Antonio TX: The Psychological Corporation (1997). Available at: http://www.amazon.com/ Wais-3-Administration-Scoring-Manual-Wechsler/dp/0158981030.

35. Keefe RSE, Harvey PD, Goldberg TE, Gold JM, Walker TM, Kennel C, et al. Norms and standardization of the Brief Assessment of Cognition in Schizophrenia (BACS). Schizophr Res (2008) 102(1-3):108-15. doi: 10.1016/ j.schres.2008.03.024

36. Sahakian BJ, Owen a M. Computerized assessment in neuropsychiatry using CANTAB: discussion paper. $R$ Soc Med (1992) 85(July):399-402. doi: 10.1177/ 014107689208500711

37. Andersson JLR, Skare S, Ashburner J. How to correct susceptibility distortions in spin-echo echo-planar images: Application to diffusion tensor imaging. Neuroimage (2003) 20(2):870-88. doi: 10.1016/S1053-8119(03)00336-7

38. Jenkinson M, Beckmann CF, Behrens TEJ, Woolrich MW, Smith SM. Fsl. Neuroimage (2012) 62(2):782-90. doi: 10.1016/j.neuroimage.2011.09.015

39. Smith SM, Jenkinson M, Johansen-Berg H, Rueckert D, Nichols TE, Mackay CE, et al. Tract-based spatial statistics: Voxelwise analysis of multi-subject diffusion data. Neuroimage (2006) 31(4):1487-505. doi: 10.1016/ j.neuroimage.2006.02.024

40. Mori S, Van Zijl P. Human white matter atlas. Am J Psychiatry (2007) 164 (July):75390. doi: 10.1176/appi.ajp.164.7.1005

41. Roalf DR, Quarmley M, Elliott MA, Satterthwaite TD, Vandekar SN, Ruparel $\mathrm{K}$, et al. The Impact of Quality Assurance Assessment on Diffusion Tensor Imaging Outcomes in a Large-Scale Population-Based Cohort. Neuroimage. (2016) 125:903-19. doi: 10.1016/j.neuroimage.2015.10.068

42. Eack SM. Cognitive Enhancement Therapy for Early-Course Schizophrenia: Effects of a. Psychiatr Serv (2009) 60:1468-76. doi: 10.1176/ps.2009.60.11.1468

43. Medalia A, Freilich B. The Neuropsychological Educational Approach to Cognitive Remediation (NEAR) Model: Practice Principles and Outcome Studies. Am J Psychiatr Rehabil (2008) 11(2):123-43. doi: 10.1080/ 15487760801963660

44. Roberts DL, David P, Combs DR. Social Cognition and Interaction Training (SCIT): Group Psychotherapy for Schizophrenia and Other Psychotic Disorders, Clinician Guide. Oxford, United Kingdom: Oxford University Press (2015).

45. Penn DL, Roberts DL, Combs D, Sterne A. Best practices: The development of the Social Cognition and Interaction Training program for schizophrenia spectrum disorders. Psychiatr Serv (2007) 58(4):449-51. doi: 10.1176/ appi.ps.58.4.449

46. Lu K, Mehrotra DV. Specification of covariance structure in longitudinal data analysis for randomized clinical trials. Stat Med (2010) 29(4):474-88. doi: $10.1002 / \operatorname{sim} .3820$

47. Jean Dunn O. Multiple Comparisons Among Means. J Am Stat Assoc (1961) 56(293):52-64. doi: 10.1080/01621459.1961.10482090

48. Hooker CI, Carol EE, Eisenstein TJ, Yin H, Lincoln SH, Tully LM, et al. A pilot study of cognitive training in clinical high risk for psychosis: Initial evidence of cognitive benefit. Schizophr Res (2014) 157(1-3):314-6. doi: 10.1016/ j.schres.2014.05.034

49. Mandl RCW, Schnack HG, Zwiers MP, van der Schaaf A, Kahn RS, Hulshoff Pol HE. Functional Diffusion Tensor Imaging: Measuring Task-Related Fractional Anisotropy Changes in the Human Brain along White Matter Tracts. PloS One (2008) 3(11):e3631. doi: 10.1371/journal.pone.0003631

50. Tavor I, Hofstetter S, Assaf Y. Micro-structural assessment of short term plasticity dynamics. Neuroimage. (2013) 81:1-7. doi: 10.1016/j.neuroimage.2013.05.050

51. Johansen-Berg H, Baptista CS, Thomas AG. Human Structural Plasticity at Record Speed. Neuron (2012) 73(6):1058-60. doi: 10.1016/j.neuron.2012.03.001

52. Schlegel A, Alexander P, Fogelson SV, Li X, Lu Z, Kohler PJ, et al. The artist emerges: Visual art learning alters neural structure and function. Neuroimage (2014) 105:440-51. doi: 10.1016/j.neuroimage.2014.11.014

53. Schlegel AA, Rudelson JJ, Tse PU. White Matter Structure Changes as Adults Learn a Second Language. J Cognit Neurosci (2012) 8):1664-70. doi: 10.1162/ jocn_a_00240
54. Taubert M, Draganski B, Anwander A, Muller K, Horstmann A, Villringer A, et al. Dynamic properties of human brain structure: learning-related changes in cortical areas and associated fiber connections 3. J Neurosci (2010) 30(1529-2401(15292401 (Electronic):11670-7. doi: 10.1523/JNEUROSCI.2567-10.2010

55. Lombroso P, Ogren M. Learning and memory, part II: molecular mechanisms of synaptic plasticity. J Am Acad Child Adolesc Psychiatry (2009) 48(1):5-9. doi: 10.1097/CHI.0b013e318190c4b3

56. Zatorre RJ, Fields RD, Johansen-Berg H. Plasticity in gray and white: neuroimaging changes in brain structure during learning. Nat Neurosci (2012) 15(4):528-36. doi: 10.1038/nn.3045

57. Wang S, Young KM. White matter plasticity in adulthood. Neuroscience (2013) 276:148-60. doi: 10.1016/j.neuroscience.2013.10.018

58. Jones DK, Knösche TR, Turner R. White matter integrity, fiber count, and other fallacies: The do's and don'ts of diffusion MRI. Neuroimage (2013) 73:239-54. doi: 10.1016/j.neuroimage.2012.06.081

59. Crocker LD, Heller W, Warren SL, O’Hare AJ, Infantolino ZP, Miller G a. Relationships among cognition, emotion, and motivation: implications for intervention and neuroplasticity in psychopathology. Front Hum Neurosci (2013) 7:261(June):261. doi: 10.3389/fnhum.2013.00261

60. Kamali A, Kramer LA, Butler IJ, Hasan KM. Diffusion tensor tractography of the somatosensory system in the human brainstem: initial findings using high isotropic spatial resolution at 3.0 T. Eur Radiol (2009) 19(6):1480-8. doi: 10.1007/s00330-009-1305-x

61. Bernard JA, Orr JM, Mittal VA. Cerebello-thalamo-cortical networks predict positive symptom progression in individuals at ultra-high risk for psychosis. NeuroImage Clin (2017) 14:622-8. doi: 10.1016/j.nicl.2017.03.001

62. Bernard JA, Dean DJ, Kent JS, Orr JM, Pelletier-Baldelli A, Lunsford-Avery JR, et al. Cerebellar networks in individuals at ultra high-risk of psychosis: Impact on postural sway and symptom severity. Hum Brain Mapp (2014) 35 (8):4064-78. doi: 10.1002/hbm.22458

63. Subramaniam K, Gill J, Fisher M, Mukherjee P, Nagarajan S, Vinogradov S. White matter microstructure predicts cognitive training-induced improvements in attention and executive functioning in schizophrenia. Schizophr Res (2018) 193:276-83. doi: 10.1016/j.schres.2017.06.062

64. Pantelis C, Wannan C, Bartholomeusz CF, Allott K, McGorry PD. Cognitive intervention in early psychosis - preserving abilities versus remediating deficits. Curr Opin Behav Sci (2015) 4:63-72. doi: 10.1016/j.cobeha.2015.02.008

Conflict of Interest: BE is part of the Advisory Board of Eli Lilly Denmark A/S, Janssen-Cilag, and Takeda Pharmaceutical Company Ltd; and has received lecture fees from Bristol-Myers Squibb, Otsuka Pharma Scandinavia AB, Eli Lilly Company, and Lundbeck Pharma A/S. BG is the leader of a Lundbeck Foundation Centre of Excellence for Clinical Intervention and Neuropsychiatric Schizophrenia Research (CINS), which is partially financed by an independent grant from the Lundbeck Foundation based on international review and partially financed by the Mental Health Services in the Capital Region of Denmark, the University of Copenhagen, and other foundations. Her group has also received a research grant from Lundbeck A/S for another independent investigator-initiated study. All grants are the property of the Mental Health Services in the Capital Region of Denmark and administrated by them. She has no other conflicts to disclose.

The remaining authors declare that the research was conducted in the absence of any commercial or financial relationships that could be construed as a potential conflict of interest.

Copyright (C) 2020 Kristensen, Ebdrup, Hjorthøj, Mandl, Raghava, Jepsen, Fagerlund, Glenthøj, Wenneberg, Krakauer, Pantelis, Glenthøj and Nordentoft. This is an openaccess article distributed under the terms of the Creative Commons Attribution License (CC BY). The use, distribution or reproduction in other forums is permitted, provided the original author(s) and the copyright owner(s) are credited and that the original publication in this journal is cited, in accordance with accepted academic practice. No use, distribution or reproduction is permitted which does not comply with these terms. 